\title{
Oncometabolite R-2 hydroxyglutarate aids the inflammatory transformation of peritumoral astrocytes
}

Vidhya Ravi ( $\nabla$ vidhya.ravi@uniklinik-freiburg.de )

University Clinic of Freiburg https://orcid.org/0000-0003-0062-2099

Simon Behringer

University Clinic of Freiburg

Kevin Joseph

University of Freiburg https://orcid.org/0000-0001-6317-8736

Luciana Haniball

University Clinic of Freiburg

Jan Kueckelhaus

University Clinic of Freiburg

Paulina Will

University Clinic of Freiburg https://orcid.org/0000-0001-5924-2432

Nicolas Neidert

University Clinic of Freiburg

Jonathan Goeldner

University Clinic of Freiburg https://orcid.org/0000-0003-2394-7312

Pamela Franco

University Clinic of Freiburg

Christian Fung

University Clinic of Freiburg

Juergen Beck

University Clinic of Freiburg

Roman Sankowski

University of Freiburg https://orcid.org/0000-0001-9215-8021

Marco Prinz

University Clinic of Freiburg

Ulrich Hofmann

University Clinic of Freiburg

Oliver Schnell

University of Freiburg

Dieter Henrik Heiland 
Medical Center - University of Freiburg https://orcid.org/0000-0002-9258-3033

\section{Article}

Keywords: glioma, anti-tumor immunity, peritumoral astrocytes

Posted Date: July 26th, 2021

DOI: https://doi.org/10.21203/rs.3.rs-720962/v1

License: (9) This work is licensed under a Creative Commons Attribution 4.0 International License. Read Full License 
3

DISCLOSURE OF CONFLICTS OF INTEREST: No potential conflicts of interest were disclosed by the authors. 
27 Reactive transformation of astrocytes in IDH wild-type glioma contribute to anti-tumor immunity and 28 support pro-oncogenic signaling. The role of intra- and peritumoral astrocytes in IDH1/2 mutated glioma, 29 a prognostically beneficial subgroup with oncogene-driven enrichment of R-2-hydroxygluterate (2-HG) 30 remains sparsely explored. Here, we characterized the transcriptomic signature of IDH1/2-mutant 31 glioma associated astrocytes and determined a unique inflammatory transformation, profoundly different 32 to astrocytes in IDH wildtype glioma patients. IDH-mutated glioma inoculation into human neocortical 33 sections or treatment with R-2-HG resulted in an oncometabolite-mediated transcriptional shift towards 34 inflammation in astrocytes. This transcriptional shift was found to be mediated by myeloid cell polarization through R-2-HG in the tumor microenvironment, which was further confirmed by selective depletion of myeloid cells. Integrative analysis of bulk RNA-sequencing of purified microglia, single-cell transcriptomics and spatially resolved transcriptomics confirmed this microglia-mediated inflammatory activation of astrocytes. This inflammatory activation is accompanied by loss of neurotransmitter homeostasis. Further, treatment of cortical sections with RH2G results in increased neuronal activity and LFP activity, pointing to the excitotoxic nature of RH2G in the neural microenvironment, with a significant loss of neurons with chronic treatment. The presented findings provide insights into the role that $\mathrm{R}-2 \mathrm{HG}$ plays in the reactive transformation of astrocytes within the IDH-mutated tumor environment and is fundamentally different that seen in IDH wildtype glioma. 


\section{INTRODUCTION}

Gliomas with a mutation in the isocitrate dehydrogenase enzyme IDH1 or 2, has been shown to primarily affect younger patients, between 20 and 40 years of age. However, these gliomas are associated with significantly better prognosis compared to IDH wild-type patients. The mutation causes an accumulation of the oncometabolite R-2-HG which replaces physiologically synthesized alpha Ketoglutarate ( $\alpha-K G)$. R-2-HG has been shown to cause epigenetic alterations in tumor cells, resulting in an accumulation of histone and DNA methylations, also referred to as the hypermethylated phenotype (Turcan et al., 2012). Recent studies suggest that this R-2-HG accumulation is not confined to the cytosol of tumor cells but is also highly enriched within the extracellular space (Fathi et al., 2016). This leads to the hypothesis that R-2-HG plays a key role in the various pathological alterations found within the microenvironment of IDH-mutated glioma. Recent reports revealed that R-2-HG plays an important role in the suppression of T-cell mediated anti-tumor immune response by disruption of the nuclear factor of activated T cells (NFAT) and polyamine biosynthesis (Bunse et al., 2018; Zhang et al., 2018). Others have described an increased inflammatory phenotype exhibited by macrophages and microglial cells within IDH-mutated gliomas (Poon et al., 2019).

The extent to which other cell types such as astrocytes and neurons are altered by R-2-HG remains poorly explored. In the context of IDH wildtype glioblastoma, we have previously reported that the reactive alterations of tumor associated astrocytes (TAA's) contribute to the evolution of an immunosuppressive microenvironment (Henrik Heiland et al., 2019) and further supports tumor growth (Wurm et al., 2019). In this work, we explored the transcriptional transformation of TAA's within the microenvironment of IDH-mutated tumors compared to wildtype glioma by means of RNA-sequencing of purified astrocytes. Using our recently established human neocortical GBM model, we were able to subsequently distinguish between the reactive transformation driven by IDH-mutated tumor cells or the oncometabolite R-2-HG. Our data revealed that R-2-HG leads to an inflammatory activation of surrounding astrocytes followed by complement activation. In addition to this astrocytic transformation, we also report that R-2-HG is able to evoke increased neuronal activity, significantly higher than seen by glutamate, which is generally enriched in IDH-wild type glioma. Our findings suggest that the role of $\mathrm{R}-2-\mathrm{HG}$ is multifaceted within the IDH-mutated glioma microenvironment, with varying effects exerted on the multitude of cell types present within the system, driving complex alterations of the tumor microenvironment and potentially contributing to the high frequency of seizures reported clinically (Chen et al., 2017). 


\section{RESULTS}

\section{Tumor-associated astrocytes in IDH-mutated glioma marked by inflammatory transformation}

We recently reported that TAA's exhibit a distinct anti-inflammatory transcriptional shift which aids in the evolution of an immunosuppressive environment in IDH1/2 wildtype glioblastoma (Henrik Heiland et al., 2019). In order to investigate the transcriptional alterations of tumor-associated astrocytes in IDH1/2 mutated glioma, we purified and transcriptionally profiled astrocytes from 9 patients with confirmed IDH1-R132H mutation, by means of RNA-sequencing. These transcriptional profiles were then compared against our previously established dataset containing transcriptomic data from astrocytes obtained from 8 non-malignant controls (Access tissue from epilepsy surgery, metastasis or glioma-IDHwildtype patients) and 7 IDH-wildtype glioblastoma, Figure 1a. Unsupervised clustering revealed a clear separation between astrocytes acquired from non-malignant specimens and tumor-associated astrocytes, Figure 1b-c. In order to further represent the astrocytes along a developmental trajectory (fetal/undifferentiated to mature/differentiated phenotype) versus their major types of reactive transformations (inflammatory to alternative transformation), we established a 2D representation as recently published (Henrik Heiland et al., 2019), Figure 1d. This 2D projection summarizes different transcriptional subtypes reported by multiple authors (Clarke et al., 2018; Escartin et al., 2021; Liddelow and Barres, 2017; Liddelow et al., 2017; Zhang et al., 2014, 2016b). This characterization reconfirmed our previous findings suggesting that reactive astrocytes in glioma further referred to as "tumorassociated astrocytes, TAA" exhibited a fetal transcriptional profile, Figure 1c. Our data suggests, that within the group of tumor-associated astrocytes, the transcriptional programs which lead to reactive transformation differ between glioma entities. Compared to TAA in IDH-wild-type glioma where Interferon gamma activation and JAK/STAT3 pathway were highly enriched, TAA's derived from IDHmutated glioma revealed an inflammatory activation, including significant up-regulation of genes coding for the factors of complement system $\left(p_{\text {adj }}<0.001\right)$, hallmark inflammatory response $\left(p_{\text {adj }}<0.001\right)$, and Tumor Necrosis Factor alpha (TNFa, $\left.p_{\text {adj }}=0.02\right)$. Immunostainings from IDH mutated tumors at the tumor edge (infiltrating border) confirmed the accumulation of complement component 3 (C3) in astrocytes, Figure 1e. To further investigate the spatial distribution of inflammatory astrocytes within IDH-mutated glioma, we extracted gene expression signatures and projected them into our recently published spatially resolved transcriptomics datasets (Ravi et al., 2021) from both IDH mutated and wildtype glioma, using a Non-negative matrix factorization (NMF) for deconvolution (Elosua et al., 2020). Our 


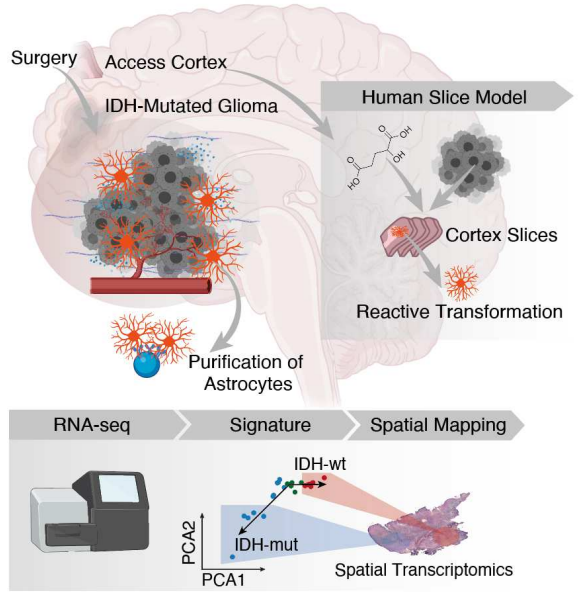

d

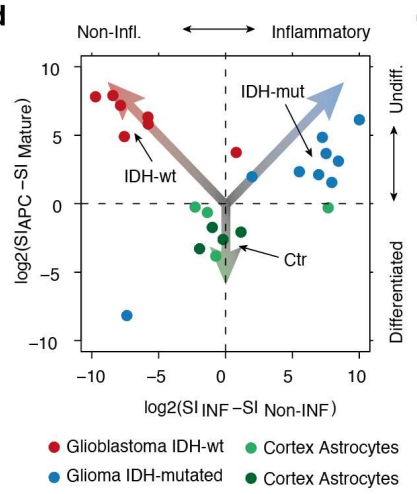

e

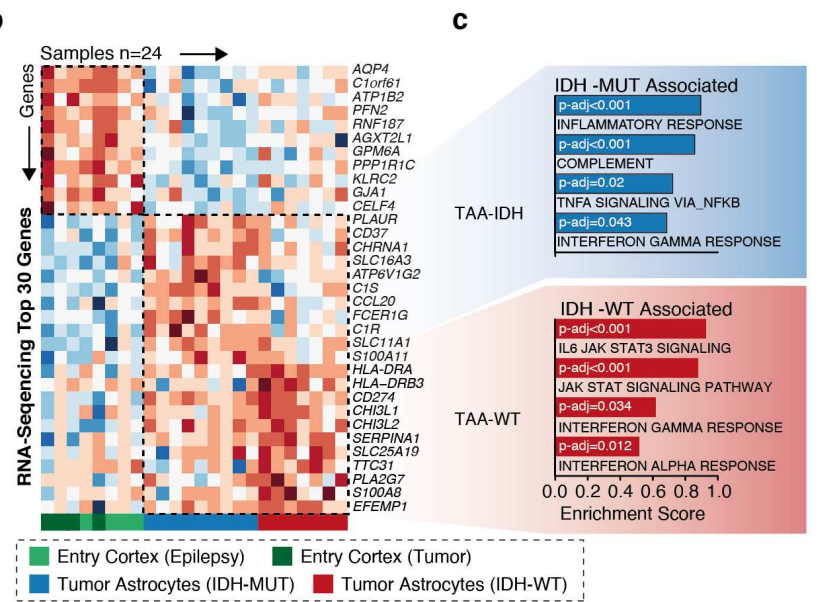

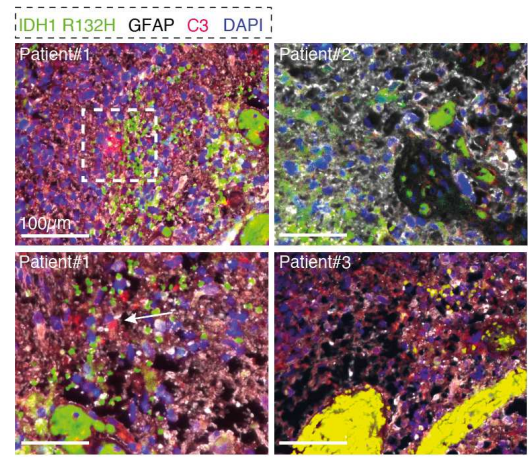

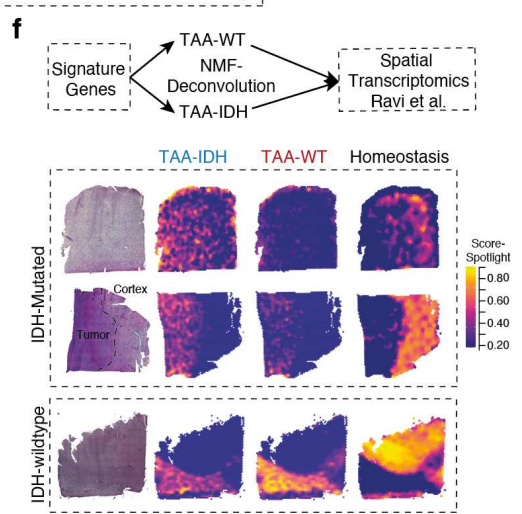

Figure 1: a) Workflow of transcriptional profiling of astrocytes from IDH mutated and wildtype tumors. B) Heatmap of transcriptional profiles. High gene expression is visualized in red, low expression in blue. The bars at the bottom display sample origin. C) Gene Set enrichment analysis of multiple pathways (MsigDB) is displayed at the right side. P-value was determined by Kolmogorov Smirnov (K-S) test and corrected by the False-Discovery Rate $\left(p_{\text {adj }}\right)$. D) 2D representation of different reactive states of astrocytes as recently established. The color depicts the sample origin. E) Immunofluorescence staining of IDHR132H mutation (tumor cells) GFAP (astrocytes and tumor cells) C3 (complement cascade) and DNA (DAPI). F) Spatial enrichment of astrocyte transformation signatures of IDH-mutated (upper) and IDH wildtype samples (bottom). Spotlight was used to integrate ranked gene signatures into space.

\section{R-2-Hydroxyglutarate activates inflammatory signaling in tumor-associated astrocytes}

118 Motivated by the findings pointing to R-2-HG mediated alterations in T cells (Bunse et al., 2018), we aimed to investigate the extent to which the reactive transformation of astrocytes is driven by the tumorcells or the oncometabolite directly. For this purpose, we used our human neocortical GBM model, either inoculated with a primary IDH-mutated cell line or treated with R-2HG, Figure 2a. Post 7 days of tumor growth, we extracted and performed RNA-sequencing from astrocytes present within the cultured sections, Figure $\mathbf{2 b}$. Unsupervised clustering and differential gene expression analysis revealed a 
124 similar inflammatory activation in astrocytes from both conditions. The reactive transformation was 125 driven by complement related genes (C3 and $C 1 Q)$ in combination with increased expression of 126 common inflammatory interleukin genes $(I L 1 A / B)$, Figure $\mathbf{2 b - c}$, which mirrors the findings in clinical 127 samples. However, to our surprise, we identified that astrocytes extracted from sections only treated 128 with R-2HG exhibited increase inflammatory activation compared to those from tumor inoculated 129 sections, as seen from our 2D representation, Figure 2d. Immunostainings confirmed the increased 130 complement activation (C3) in astrocytes from sections treated with R-2HG, Figure 2e. In summary, 131 these findings suggest that the oncometabolite R--2-HG contributes directly to the inflammatory 132 transformation of astrocytes.

a

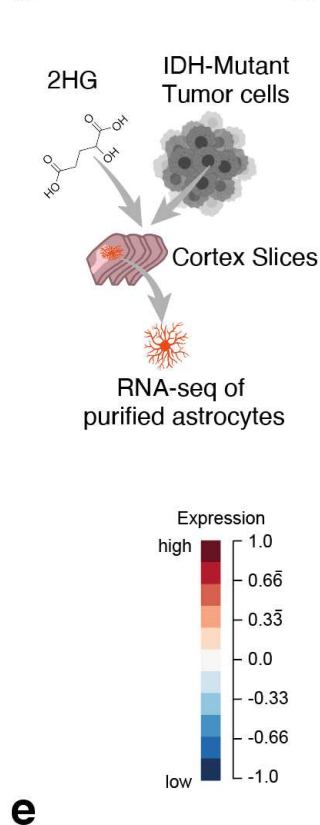

b

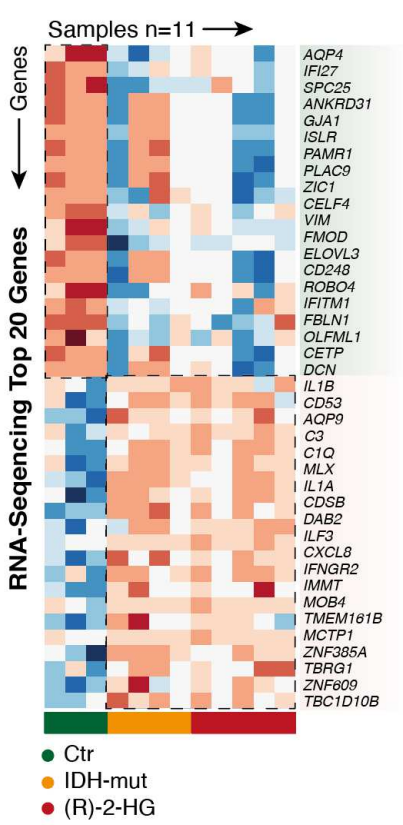

C
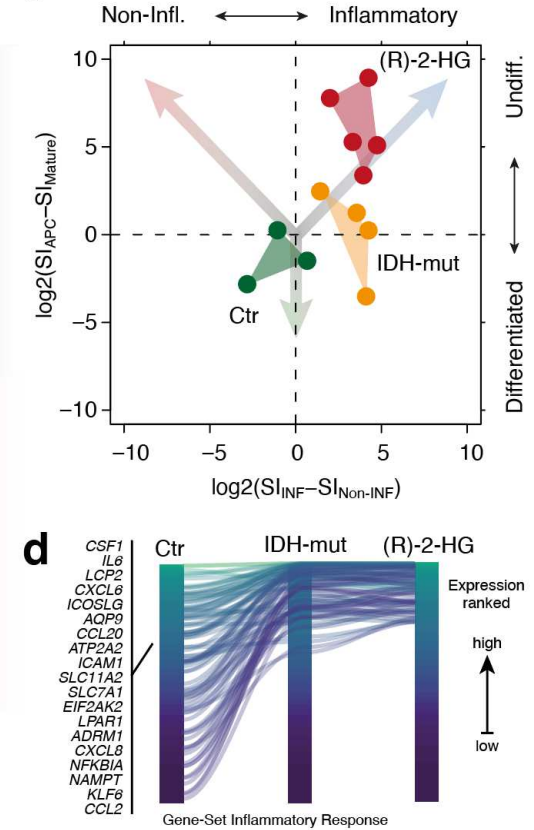

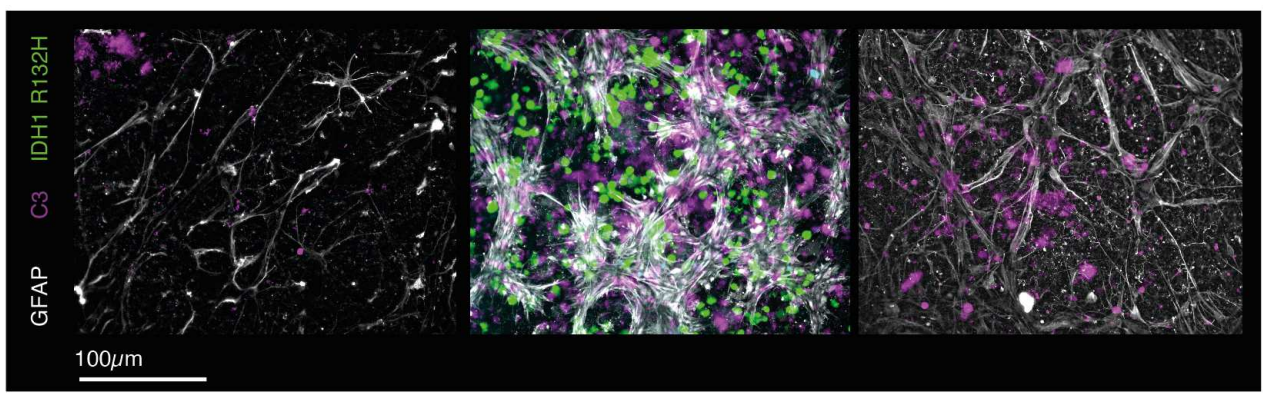

$\perp 40$

Figure 2: a) Workflow of transcriptional profiling of astrocytes from slices with IDH-mutated tumor injection or R--2HG treatment.

148 B) Heatmap of transcriptional profiles. High gene expression is visualized in red, low expression in blue. The bars at the bottom

149 reveal the sample origin. C) $2 D$ representation of different reactive states of astrocytes as recently established. The color determines the sample origin. D) River plot of gene expression changes (Inflammatory response) in different conditions. E) Immunofluorescence staining of IDHR132H mutation (tumor cells) GFAP (astrocytes and tumor cells) C3 (complement cascade). 


\section{R-2-Hydroxyglutarate induces reactive activation through myeloid cells}

153 We and others have previously reported that the inflammatory transformation of astrocytes is 154 predominantly driven by myeloid cells (Clarke et al., 2018; Henrik Heiland et al., 2019; Liddelow et al., $1552017,2020)$. To validate if this holds true for R-2HG mediated astrocytic transformation, we used our 156 previously established chemical based ex-vivo myeloid cell depletion model (Ravi et al., 2019). For a 157 positive control of inflammatory stimulation, we used lipopolysaccharide (LPS) which is known to drive 158 an inflammatory reactive phenotype of astrocytes mediated by microglial activation (Clarke et al., 2018; 159 Liddelow et al., 2017; Yun et al., 2018). Sections that underwent myeloid cell depletion were either 160 inoculated with IDH-mutated GBM or were treated with R-2HG / LPS, Figure 3a. The absence of IBA1 ${ }^{+}$ myeloid cells was validated by means of immunofluorescence post depletion for $72 \mathrm{~h}$, Figure $\mathbf{3 b}$.

162 To determine the effect that each condition had on astrocytic activation, we estimated the number of

$163 \mathrm{C}^{+}$astrocytes, Figure 3c. We observed a significant increase in $\mathrm{C}^{+}$astrocytes in all treatment conditions, with the highest number of $\mathrm{C}^{+}$astrocytes observed due to R-2HG treatment. No significant increase in $\mathrm{C}^{+}$astrocytes were observed when myeloid depletion was carried out, Figure $3 \mathrm{c}$. These results confirm that astrocytic transformation due to R-2HG is primarily mediated by myeloid cells in the tumor microenvironment. 


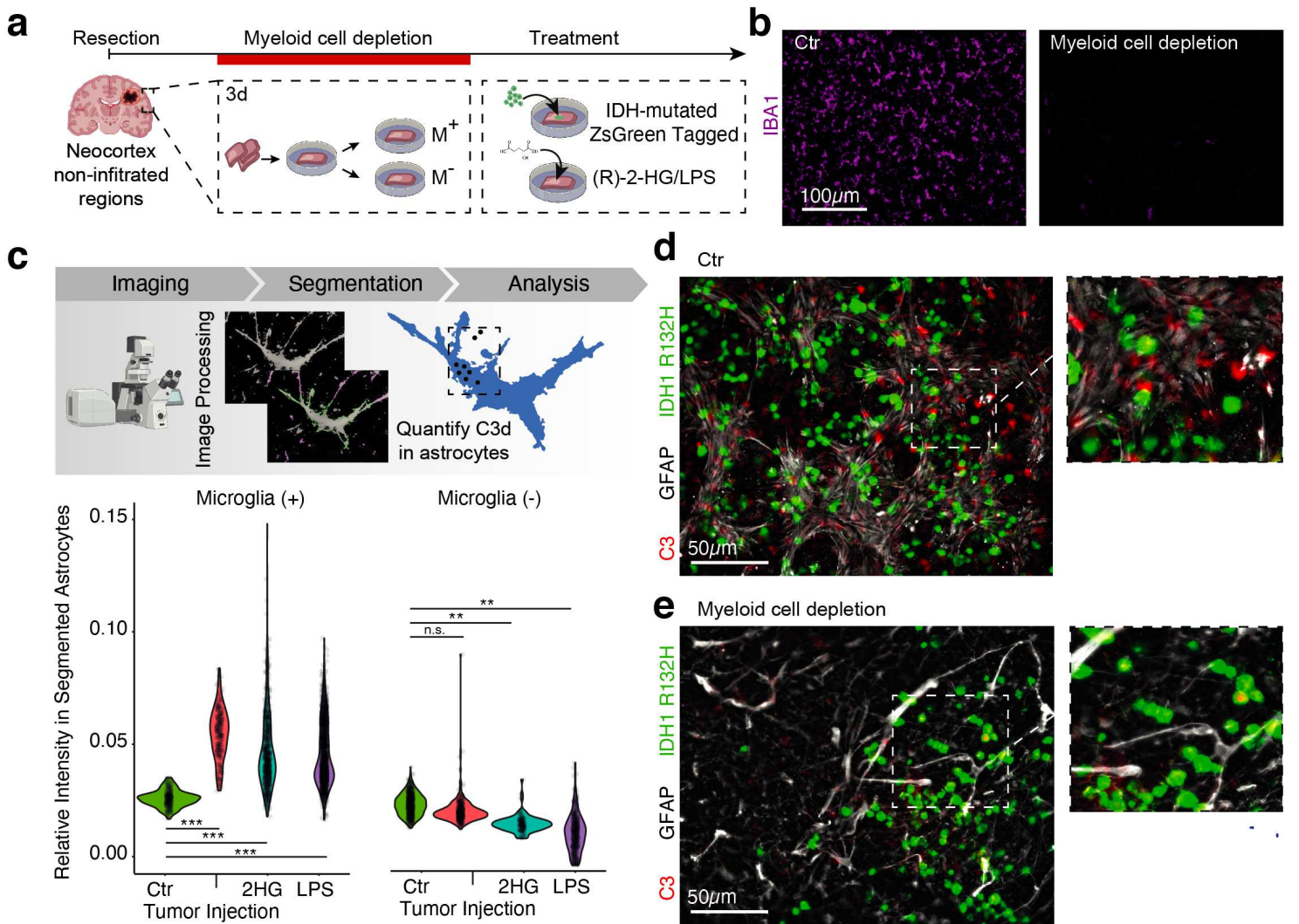

Figure 3: a) Workflow of the slice model with myeloid cell depletion and LPS/2HG stimulation. B) Immunofluorescence image of ctr and microglia/myeloid cell depletion after 3 days clodronate treatment with IBA1. A significant reduction of IBA ${ }^{+}$cells are demonstrated ( $p<0.001)$, data not shown. C) Imaging analysis pipeline combining trained segmentation algorithm (ilastik) to quantify the inflammatory marker (C3) in astrocytes from different conditions. Quantification demonstrated at the bottom. Alpha level was determined by Wilcoxon-Sum Rank statistics and illustrated by ${ }^{*} p<0.01,{ }^{* *} p<0.001$ and ${ }^{* * *} p<0.0001$. Each points represents single cells. D) Immunofluorescence image of ctr and microglia/myeloid cell depletion with C3 (red), GFAP (white) and tumor cells in green. Cellular shapes determined by the segmentation are illustrated at the bottom right.

Inflammatory myeloid cells drive inflammatory activation of astrocytes.

182 In order to comprehensively interrogate the transcriptional heterogeneity exhibited by myeloid cells 183 within both the IDH wildtype and IDH mutated environments, we analyzed a recently published publicly 184 available dataset (Friedrich et al., 2021), Supp. Figure 1a. Unsupervised clustering to decipher major 185 regulatory programs revealed 5 major clusters, further summarized into 3 modules, based on partial 186 similarity, Supp. Figure 1b. The first module represents homeostatic microglia with a large percentage 187 of microglia from non-malignant samples. The remaining 2 modules show distinct activation patterns, 188 namely non-inflammatory and inflammatory. The non-inflammatory activation pattern is marked by 189 interferon gamma activation and expression of CD163 and TGFB, Supp. Figure 1c. This module 190 contained most of the microglial cells from IDH wild type tumors. The inflammatory module is marked 191 by expression of common inflammatory cytokines such as IL1B and TNF and was composed of microglia 
derived from IDH-mutated tumors, which is in line with our findings from the cortical GBM model, Supp.

194 In order to confirm microglia activation patterns, we transcriptionally profiled purified myeloid cells $195\left(\mathrm{CD}^{+} 5^{+}\right)$from our human GBM model inoculated with either IDH mutated or wildtype glioma cells. The expression profiles were then projected into the PCA space of the single-cell dataset to align the myeloid activation from our cortical GBM model, Figure 4a. This approach helped us confirm the inflammatory character of myeloid cells from IDH-mutated vs IDH-wild type tumor microenvironment. In order to correlate the estimated co-activation of astrocytes and myeloid cells, spatial correlation was carried out by inferring the likeliness of neighborhood, Figure $\mathbf{4 b - c}$. Our data suggests that astrocytic and myeloid activation was spatially co-localized only within IDH mutated tumor environment, Figure 4d.

a

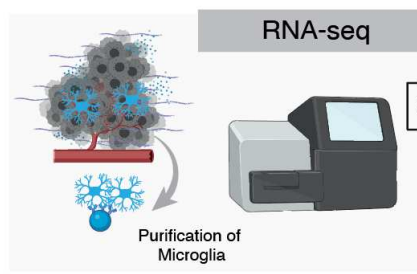

b

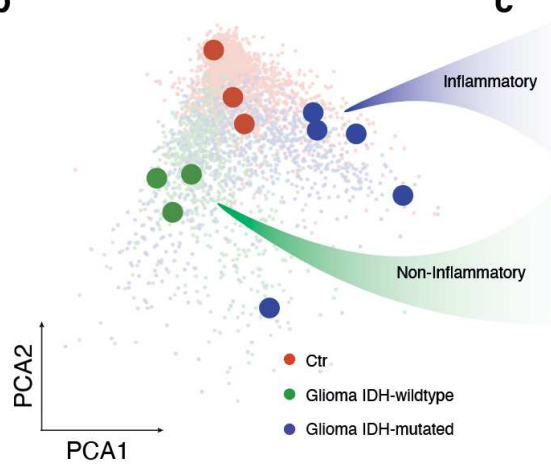

h

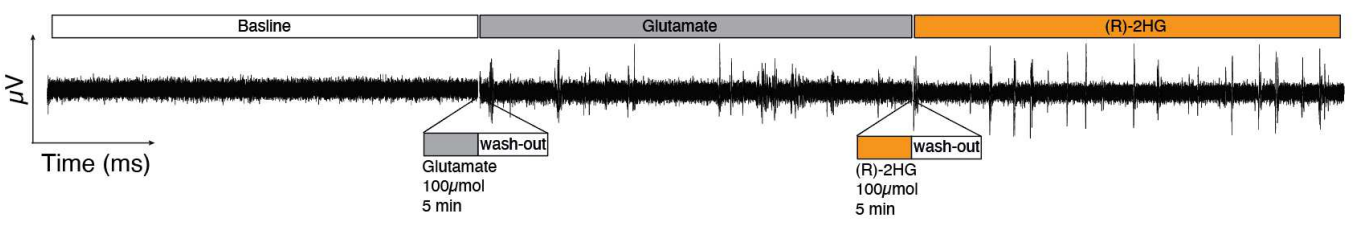
a RNN trained on multidimensional PCA space was trained to predict the position of purified microglia (bulk-seq). c) For in-depth characterization of the spatial context, we estimated the spatial localization as explained in Figure 1. D) To estimate the co- 
of IDH-specific signatures of astrocytes and microglia cells. e) Immunofluorescence image of slices with tumor injection and R-2HG treatment illustrate a loss of NeuN positive cells in both conditions. f) Quantification of cell counts demonstrate a significant loss of NeuN pos. cells compared to ctr slices. Alpha level was determined by Wilcoxon-Sum Rank statistics and illustrated by * $p<0.05,{ }^{* *} p<0.01$. Points in the bar plot represents biological replicates. $g$ ) Barplots of electrophysiology quantification of spikes in various conditions h) Example traces (MEA) from slices with Baseline, Glutamate and R-2HG stimulation.

\section{R-2-Hydroxygluterate mediates neuronal excitotoxicity}

213 Since R-2HG drives reactive transformation of astrocytes, there is a loss of normal homeostatic function

214 (Escartin et al., 2021; Li et al., 2019), as seen in our data, Supp Fig. 1e. This reactive transformation is 215 independent of the age of the specimens, Supp Fig. 1f. Since the reactive transformation has been 216 shown to lead to a loss of homeostasis, which should be reflected in neurotransmitter profiles, we carried 217 out metabolic profiling (LC-MS) from culture medium collected from both IDH-mutated glioma inoculated 218 and R-2HG treated sections. Our findings showed that there is no significant increase in extracellular 219 glutamate in IDH-mut GBM inoculated section, whereas treatment with R-2HG led to elevated levels of 220 extracellular glutamate $(p=0.005)$, Supp Fig $\mathbf{2 a}$, which is generally neurotoxic. Imaging results 221 complement these results, where we observed a significant reduction in neuronal cells in sections that 222 are treated with R-2HG, compared to IDH inoculated and control sections, Fig. 4 e-f. In addition to the 223 role that plays in neuroinflammation, the chemical structure of R-2HG is highly similar to glutamate, 224 which could potentially induce excitotoxicity by means of increased glutamatergic activation. To 225 determine if neuronal activity can be influenced by secreted R-2HG, we exposed cultured sections to 226 both glutamate $(100 \mu \mathrm{M})$ and R-2HG $(50 \mu \mathrm{M})$. Glutamate exposure resulted in a significant increase in 227 spiking events, with increased spontaneous LFP activity, Figure $\mathbf{4 g}$. Further exposure of the same 228 sections with R-2HG post washout resulted in further increase in both spike rates in addition to the 229 frequency and amplitude of LFP events. These results suggest that chronic exposure to R-2HG could result in excitotoxicity, culminating in neuronal death, Figure $\mathbf{4 h}$. 


\section{DISCUSSION}

IDH1/2 mutated glioma are a distinct group of brain tumors that differ from IDH-wildtype glioma in several aspects besides clinical prognosis (Han and Batchelor, 2017; Huang et al., 2019; Waitkus et al., 2016).

234 A crucial factor for the growth and invasive behavior of glioma is the tumor microenvironment and its supportive or inhibitory impact on the tumor. We have demonstrated that both astrocytes and microglia engage in non-inflammatory transformation in IDH wild-type gliomas which promote an immunosuppressive milieu (Henrik Heiland et al., 2019). Consequently, anti-tumor immunity is suppressed and concurrently prooncogenic stimuli from myeloid cells and astrocytes promote tumor growth (Bunse et al., 2018; Zhang et al., 2016a). Some of these prooncogenic effects have been shown to be mediated by the oncometabolite R-2HG in IDH-mutated tumors, Most recently, the impact of R$2 \mathrm{HG}$ on $\mathrm{T}$ cells was investigated, confirming that the $\mathrm{R}-2 \mathrm{HG}$ has a major impact on various cells of the IDH mutated tumor microenvironment (Friedrich et al., 2021). Motivated by this evidence, we explored the role of R-2HG on glial activation. In this study, we made use of a human neocortical slice model which was established recently to study the tumor microenvironment without interspecies bias. Since there are considerable differences within species with regards to the reactive transformation of astrocytes (Ravi et al., 2019), we believe that the human model has a decisive advantage. Our data suggests that both IDH tumor inoculation into tissue sections and R-2HG treatment were able to activate inflammatory transcriptional programs in astrocytes, mediated by the presence of microglia. Further, by spatially mapping the transcriptomic profiles of purified microglia, we were able to confirm that microglia also demonstrate inflammatory activation in IDH mutated glioma. This inflammatory astrocyte transformation is associated with a loss of neurotransmitter homeostasis, which is seen in our data with disrupted levels of glutamate in the treated sections, as has been previously reported in IDH mutated tumors (Nagashima et al., 2016). Additionally, R-H2G increased neuronal spiking rate in, pointing to potential excitotoxicity. This was shown to be true when the sections were incubated with $50 \mu \mathrm{m}$ R-2HG resulted in a significant reduction of neurons compared to controls. to IDH wild-type glioma. 


\section{Acknowledgement}

$261 \mathrm{DHH}$ is funded by the Else-Kröner Memorial Scholarship, JK is funded by the German Cancer Aid 262 (Krebshilfe project numbe0r 70113801). VR, KJ and UGH funded by BMBF (Bundes Ministerium für 263 Bildung und Forschung, Project number: FMT 13GW0230A), We thank Dietmar Pfeifer for his helpful 264 advice. We thank Biorender.com.

265

\section{Author contributions}

267 The study was designed and coordinated by DHH and VMR. Purification of astrocytes and FACS was 268 performed by SPB, JG and NN. FACS analysis was checked by MF. Mass Spectrometric analysis was 269 performed by LH. Tissue material was provided by OS, PF and CF. Neuropathological analysis was 270 performed by RS and MP. Organotypic slice culture and Immunostainings was performed by VMR and 271 KJ. Spatial transcriptomics data collection was performed by VMR and PW. Electrophysiology recording 272 was performed by KJ. Bioinformatical analysis was performed by DHH and JK. Manuscript writing editing 273 and figure design was performed by $\mathrm{DHH}, \mathrm{VMR}, \mathrm{KJ}, \mathrm{PF}$. UGH, OS and JB helped oversee the project. 274

\section{Conflict of interests}

276 No potential conflicts of interest were disclosed by the authors.

277 


\section{Bibliography}

Behringer, S., Wingert, V., Oria, V., Schumann, A., Grünert, S., Cieslar-Pobuda, A., Kölker, S., Lederer, A.-K., Jacobsen, D.W., Staerk, J., et al. (2019). Targeted metabolic profiling of methionine cycle metabolites and redox thiol pools in mammalian plasma, cells and urine. Metabolites 9 .

Bunse, L., Pusch, S., Bunse, T., Sahm, F., Sanghvi, K., Friedrich, M., Alansary, D., Sonner, J.K., Green, E., Deumelandt, K., et al. (2018). Suppression of antitumor T cell immunity by the oncometabolite (R)-2-hydroxyglutarate. Nat. Med. 24, 1192-1203.

Chen, H., Judkins, J., Thomas, C., Wu, M., Khoury, L., Benjamin, C.G., Pacione, D., Golfinos, J.G., Kumthekar, P., Ghamsari, F., et al. (2017). Mutant IDH1 and seizures in patients with glioma. Neurology $88,1805-1813$.

Clarke, L.E., Liddelow, S.A., Chakraborty, C., Münch, A.E., Heiman, M., and Barres, B.A. (2018). Normal aging induces A1-like astrocyte reactivity. Proc. Natl. Acad. Sci. USA 115, E1896-E1905.

Elosua, M., Nieto, P., Mereu, E., Gut, I., and Heyn, H. (2020). SPOTlight:Seeded NMF regression to Deconvolute Spatial Transcriptomics Spots with Single-Cell Transcriptomes. BioRxiv.

Escartin, C., Galea, E., Lakatos, A., O’Callaghan, J.P., Petzold, G.C., Serrano-Pozo, A., Steinhäuser, C., Volterra, A., Carmignoto, G., Agarwal, A., et al. (2021). Reactive astrocyte nomenclature, definitions, and future directions. Nat. Neurosci. 24, 312-325.

Fathi, A.T., Nahed, B.V., Wander, S.A., Iafrate, A.J., Borger, D.R., Hu, R., Thabet, A., Cahill, D.P., Perry, A.M., Joseph, C.P., et al. (2016). Elevation of Urinary 2-Hydroxyglutarate in IDH-Mutant Glioma. Oncologist 21, 214-219.

Friedrich, M., Sankowski, R., Bunse, L., Kilian, M., Green, E., Ramallo Guevara, C., Pusch, S., Poschet, G., Sanghvi, K., Hahn, M., et al. (2021). Tryptophan metabolism drives dynamic immunosuppressive myeloid states in IDH-mutant gliomas. Nat. Cancer.

Han, C.H., and Batchelor, T.T. (2017). Isocitrate dehydrogenase mutation as a therapeutic target in gliomas. Chin. Clin. Oncol. 6, 33.

Hastie, T.J., Narasimhan, B., Tibshirani, R.J., and Chu, G. Predictive analysis of microarrays. 1-41 (2002).

Henrik Heiland, D., Ravi, V.M., Behringer, S.P., Frenking, J.H., Wurm, J., Joseph, K., Garrelfs, N.W.C., Strähle, J., Heynckes, S., Grauvogel, J., et al. (2019). Tumor-associated reactive astrocytes aid the evolution of immunosuppressive environment in glioblastoma. Nat. Commun. 10, 2541.

Huang, J., Yu, J., Tu, L., Huang, N., Li, H., and Luo, Y. (2019). Isocitrate dehydrogenase mutations in glioma: from basic discovery to therapeutics development. Front. Oncol. 9, 506. Li, K., Li, J., Zheng, J., and Qin, S. (2019). Reactive astrocytes in neurodegenerative diseases. Aging Dis 10, 664-675.

Liddelow, S.A., and Barres, B.A. (2017). Reactive astrocytes: production, function, and therapeutic potential. Immunity 46, 957-967.

Liddelow, S.A., Guttenplan, K.A., Clarke, L.E., Bennett, F.C., Bohlen, C.J., Schirmer, L., Bennett, M.L., Münch, A.E., Chung, W.-S., Peterson, T.C., et al. (2017). Neurotoxic reactive astrocytes are induced by activated microglia. Nature 541, 481-487.

Liddelow, S.A., Marsh, S.E., and Stevens, B. (2020). Microglia and Astrocytes in Disease: Dynamic Duo or Partners in Crime? Trends Immunol.

Love, M.I., Huber, W., and Anders, S. (2014). Moderated estimation of fold change and dispersion for RNA-seq data with DESeq2. Genome Biol. 15, 550.

Maier, J., Ravi, V., Kueckelhaus, J., Behringer, S., Garrelfs, N., Will, P., Sun, N., Ehr, J., Goeldner, J., Pfeifer, D., et al. (2021). Inhibition of Metabotropic Glutamate Receptor III facilitates sensitization to alkylating chemotherapeutics in Glioblastoma. Res. Sq. McCullagh, P. (1984). Generalized linear models. Eur. J. Oper. Res. 16, 285-292. 
Nagashima, H., Tanaka, K., Sasayama, T., Irino, Y., Sato, N., Takeuchi, Y., Kyotani, K., Mukasa, A., Mizukawa, K., Sakata, J., et al. (2016). Diagnostic value of glutamate with 2hydroxyglutarate in magnetic resonance spectroscopy for IDH1 mutant glioma. Neuro. Oncol. $18,1559-1568$. Poon, C.C., Gordon, P.M.K., Liu, K., Yang, R., Sarkar, S., Mirzaei, R., Ahmad, S.T., Hughes, human IDH-mutant and -wild type glioblastoma. Oncotarget 10, 3129-3143. Ravi, V.M., Joseph, K., Wurm, J., Behringer, S., Garrelfs, N., Errico, P. d', Naseri, Y., Franco, P., Meyer-Luehmann, M., Sankowski, R., et al. (2019). Human organotypic brain slice culture: a novel framework for environmental research in neuro-oncology. Life Sci. Alliance 2, e201900305.

Ravi, V.M., Will, P., Kuekelhaus, J., Sun, N., Joseph, K., Salie, H., von Ehr, J., Vollmer, L., Benotmane, J.K., Neidert, N., et al. (2021). Spatiotemporal heterogeneity of glioblastoma is dictated by microenvironmental interference. BioRxiv.

Subramanian, A., Tamayo, P., Mootha, V.K., Mukherjee, S., Ebert, B.L., Gillette, M.A., Paulovich, A., Pomeroy, S.L., Golub, T.R., Lander, E.S., et al. (2005). Gene set enrichment analysis: a knowledge-based approach for interpreting genome-wide expression profiles. Proc. Natl. Acad. Sci. USA 102, 15545-15550.

Turcan, S., Rohle, D., Goenka, A., Walsh, L.A., Fang, F., Yilmaz, E., Campos, C., Fabius, A.W.M., Lu, C., Ward, P.S., et al. (2012). IDH1 mutation is sufficient to establish the glioma hypermethylator phenotype. Nature 483, 479-483.

Van der Maaten, L., and Hinton, G. (2008). Visualizing data using t-SNE. Journal of Machine Learning Research.

Waitkus, M.S., Diplas, B.H., and Yan, H. (2016). Isocitrate dehydrogenase mutations in gliomas. Neuro. Oncol. 18, 16-26.

Wilkerson, M.D., and Hayes, D.N. (2010). ConsensusClusterPlus: a class discovery tool with confidence assessments and item tracking. Bioinformatics 26, 1572-1573.

Wurm, J., Behringer, S.P., Ravi, V.M., Joseph, K., Neidert, N., Maier, J.P., Doria-Medina, R., Follo, M., Delev, D., Pfeifer, D., et al. (2019). Astrogliosis Releases Pro-Oncogenic Chitinase 3-Like 1 Causing MAPK Signaling in Glioblastoma. Cancers (Basel) 11.

Xu, H., Wang, Z., Zhu, L., Sui, Z., Bi, W., Liu, R., Bi, K., and Li, Q. (2018). Targeted Neurotransmitters Profiling Identifies Metabolic Signatures in Rat Brain by LC-MS/MS: Application in Insomnia, Depression and Alzheimer's Disease. Molecules 23. Yun, S.P., Kam, T.-I., Panicker, N., Kim, S., Oh, Y., Park, J.-S., Kwon, S.-H., Park, Y.J., Karuppagounder, S.S., Park, H., et al. (2018). Block of A1 astrocyte conversion by microglia is neuroprotective in models of Parkinson's disease. Nat. Med. 24, 931-938.

Zhang, L., Sorensen, M.D., Kristensen, B.W., Reifenberger, G., McIntyre, T.M., and Lin, F. (2018). D-2-Hydroxyglutarate Is an Intercellular Mediator in IDH-Mutant Gliomas Inhibiting Complement and T Cells. Clin. Cancer Res. 24, 5381-5391.

Zhang, X., Rao, A., Sette, P., Deibert, C., Pomerantz, A., Kim, W.J., Kohanbash, G., Chang, Y., Park, Y., Engh, J., et al. (2016a). IDH mutant gliomas escape natural killer cell immune surveillance by downregulation of NKG2D ligand expression. Neuro. Oncol. 18, 1402-1412. Zhang, Y., Chen, K., Sloan, S.A., Bennett, M.L., Scholze, A.R., O’Keeffe, S., Phatnani, H.P., Guarnieri, P., Caneda, C., Ruderisch, N., et al. (2014). An RNA-sequencing transcriptome and splicing database of glia, neurons, and vascular cells of the cerebral cortex. J. Neurosci. 34, 11929-11947.

Zhang, Y., Sloan, S.A., Clarke, L.E., Caneda, C., Plaza, C.A., Blumenthal, P.D., Vogel, H., Steinberg, G.K., Edwards, M.S.B., Li, G., et al. (2016b). Purification and Characterization of Progenitor and Mature Human Astrocytes Reveals Transcriptional and Functional Differences with Mouse. Neuron 89, 37-53.

Supplementary Figure 1: 
a

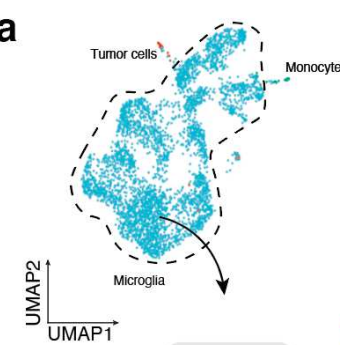

b
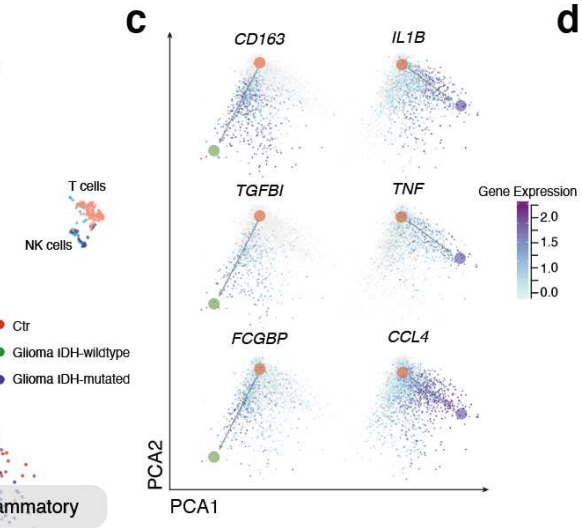

Inflammatory

- Giloma IDH-wildtype - Giloma IOH-mutated

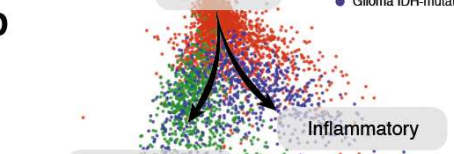

Non-Inflammatory

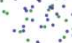

$\underset{\text { PCA1 }}{\stackrel{\text { Not }}{\longrightarrow}}$

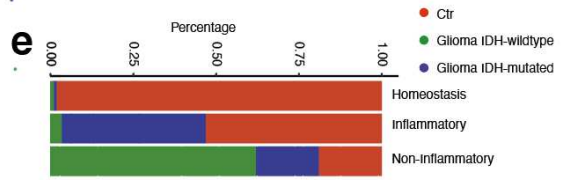

d

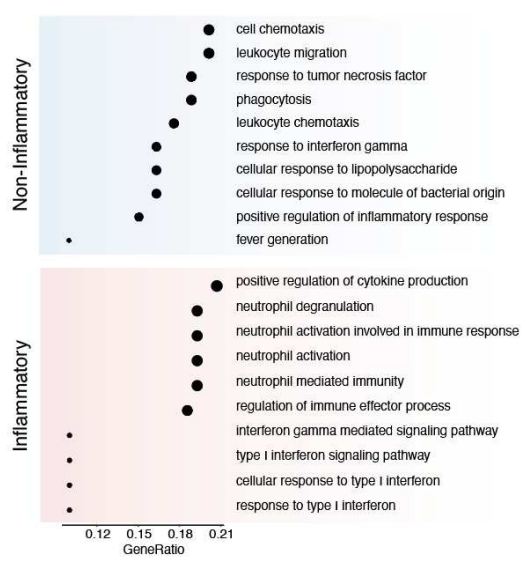

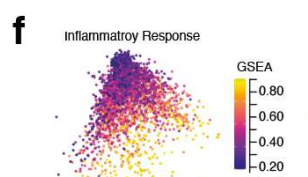

Supplementary Figure 1: a) UMAP reduction showing transcriptional heterogeneity in myeloid cells using a single cell dataset 
Supplementary Figure 2:

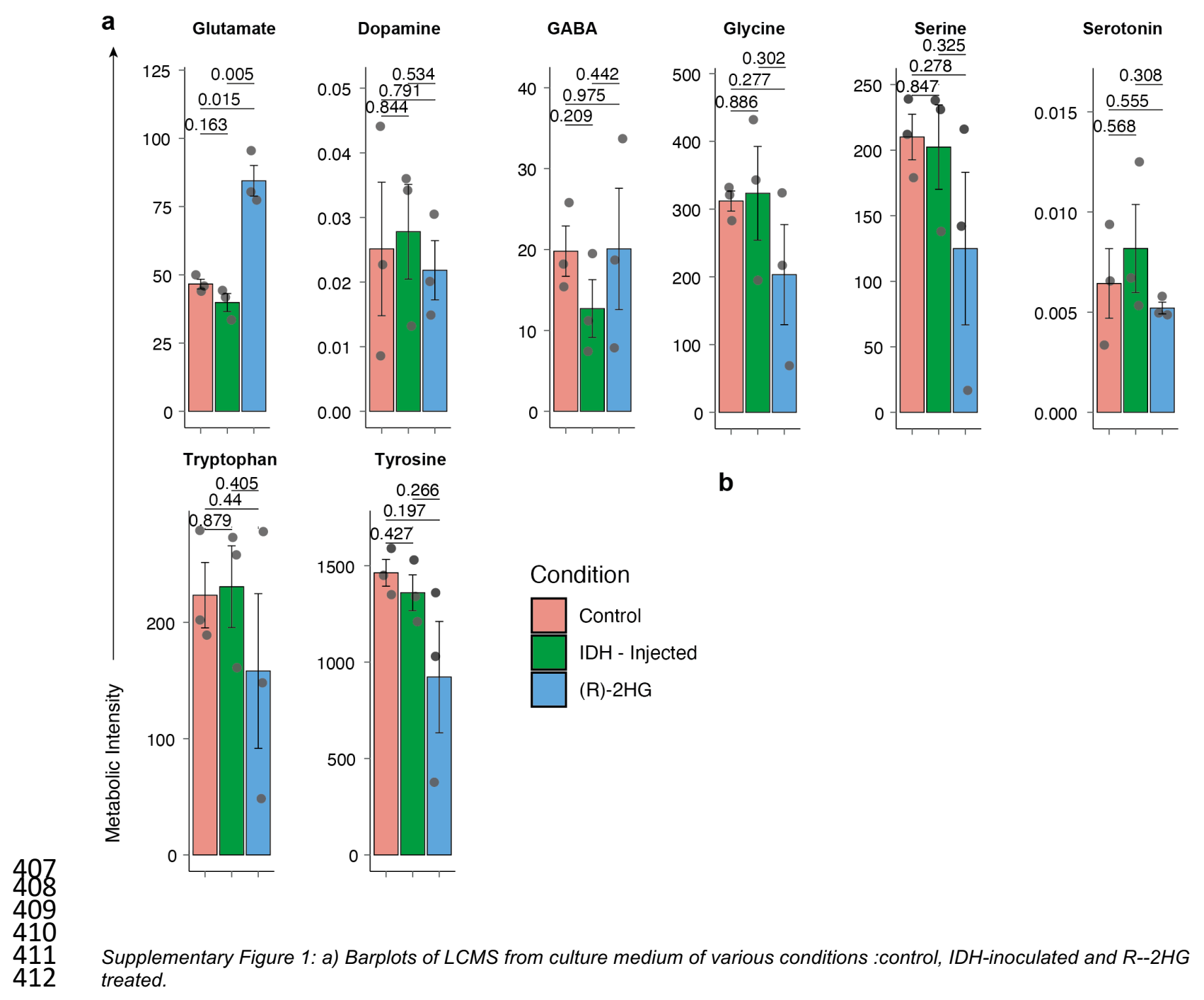




\section{Ethical Approval and Resource Sharing}

416 The local ethics committee of the University of Freiburg approved data evaluation, imaging procedures 417 and experimental design (protocol 100020/09 and 472/15_160880). The methods were carried out in 418 accordance with the approved guidelines, with written informed consent obtained from all subjects. The 419 studies were approved by an institutional review board.

Cortical GBM model

421 Tissue preparation: Capillaries and visibly damaged tissue were dissected away from donor tissue block 422 submerged in preparation medium containing: Hibernate medium supplemented with $13 \mathrm{mM}$ Glucose,

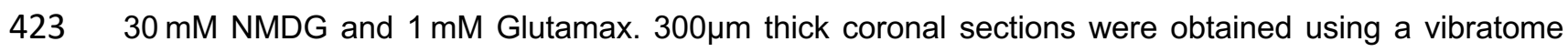
424 (VT1200, Leica, Germany) which were retained in Carbogen $\left(95 \% \mathrm{O}_{2} / 5 \% \mathrm{CO}_{2}\right.$ ) bubbled ice-cold 425 preparation medium for $10 \mathrm{~min}$ before plating to help removal of dead/damaged cells at the section 426 surfaces. 3-4 sections were gathered per insert (PICMORG50, Merck-Millipore, USA). The cultured 427 sections were maintained in growth medium containing Neurobasal (L- Glutamine) supplemented with $2 \%$ serum free B-27, 2\% Anti- Anti, 13 mM D+ Glucose, 1 mM MgSO4, 15 mM HEPES (Sigma, H0887), and $2 \mathrm{mM}$ Glutamax at $5 \% \mathrm{CO}_{2}$ and $37^{\circ} \mathrm{C}$. The culture medium was replaced at $24 \mathrm{~h}$ post plating, and then every $48 \mathrm{~h}$ for the course of the culture period. Culture medium was collected and stored at $-20^{\circ} \mathrm{C}$ for further LC-MS measurements.

\section{$\underline{\text { Tumor cell inoculation }}$}

434 The injected tumor cell line is a patient derived, confirmed IDH-mutated GBM cell line. It was harvested 435 and cultivated exclusively in the Universitätstklinik Freiburg since late 2018. Cell were cultured in serum436 free conditions as described previously, Heiland et al. 2018. For mutation and growth analysis 437 (electrophysiology) cells were plated on laminin coated dishes as described in previous manuscripts. In 438 line with previous descriptions the cell line has shown a relatively slow growth rate. In order to monitor 439 tumor invasion and proliferation profile, cells had to be fluorescently labeled using Thermo fishers Cell 440 trace CFSE dye (Thermo Fisher Scientific, Waltham, Massachusetts, USA) prior to injection. Therefore, 441 cultured cells were trypsinized, centrifuged and counted. Approximately one million cells were then 442 resuspended in $1 \mathrm{~mL}$ of PBS. $1 \mu \mathrm{L}$ of Celltrace CFSE dye is added. Cells were then incubated for 20 443 minutes at $37^{\circ} \mathrm{C}$. In order to eliminate any unbound dye $5 \mathrm{~mL}$ of culture medium containing at least $1 \%$ 444 of serum is added after incubation. Cells are then incubated 5 minutes and subsequently spun down. 445 Cells were then resuspended in MEM media at 40,000 cells $/ \mu \mathrm{L}$. Cells were used immediately for 446 injection onto tissue slices. A $10 \mu \mathrm{L}$ Hamilton syringe was used to inject $1 \mu \mathrm{L}$ into the white matter portion 447 of the slice culture.

448 Slices with and without injected tumor cells were incubated at $37^{\circ} \mathrm{C}, 5 \% \mathrm{CO}_{2}$ for 7 days and fresh culture 449 medium was added every 2 days. And, slices with R-2HG treatment were incubated in growth medium 450 with a final concentration of $50 \mu \mathrm{M}$ D-2hydroxyglutaric acid (Merck, Darmstadt Germany) for 7 days. 451 After 7 days of culture, slices were harvested and processed to either single cell suspensions in the 
above-described manner or fixed in 4\% PFA for further immunofluorescence-based staining.

453

454

455

456

457

458

459

460

461

462

463

464

465

466

467

468

469

470

471

472

473

474

475

476

477

478

479

480

481

482

483

484

485

486

487

488

489

490

491

492

\section{Isolation of cells of interest from the donor tissue/slice model}

Viable single-cell suspensions from primary tumor tissue for subsequent MACs-sorting were generated by using a combined enzymatic-mechanical dissociation approach. Tissue was collected from surgery in phosphate buffered saline and placed in a sterile cell culture bench. All steps were performed under sterile conditions After final dissociation the cell solution is passed through a 40um strainer (BD Falcon \#352340). The strainer is rinsed with $10 \mathrm{ml}$ of cold HBSS. After centrifugation $\left(310 \mathrm{~g}, 4^{\circ} \mathrm{C} ; 6 \mathrm{~min}\right)$, the remaining pellet is immediately processed for myelin removal via the Miltenyi debris removal kit (Miltenyi Biotec, Bergisch Gladbach, Germany). Therefore, the pellet is resuspended in the appropriate amount of debris removal solution and PBS given by the manufacturers protocol. Suspensions are then centrifuged at $300 \mathrm{xg}$ for 10 minutes in a swing bucket centrifuge, in order to minimize cell loss. After successful myelin removal cells were counted and either frozen at $-80^{\circ} \mathrm{C}$ or used in magnetic separation immediately.

We then made use of magnetic cell isolation, based on nano immuno-magnetic beads, combined with selective columns (MACS; Militenyi Biotec). Astrocytes were tagged with $20 \mu \mathrm{l}$ of biotin labelled antiGLAST primary antibody (ACSA-1, Militenyi Biotec, Germany), with $10^{7}$ cells used as the input. Post incubation, we performed magnetic labelling with $20 \mu \mathrm{m}$ beads. The tagged astrocytes were then isolated using a MACS column (MS Columns, Militenyi Biotec) and washed. The resulting output was collected directly into Qiazol (79306, Qiagen, Germany) followed directly by RNA extraction and validation using a NanoDrop (2000c, Thermofischer Scientific, USA) Spectrophotometer.

\section{Clodronate depletion model}

Selective depletion of microglia in organotypic brain sections from IDH donors was carried out by supplementation of culture medium with $1 \mathrm{mg} / \mathrm{ml}$ Clodronate (Sigma, D4434) for $72 \mathrm{~h}$. Subsequently, the slice cultures were rinsed with growth medium to wash away any debris. Depletion success was monitored by means of immunofluorescence.

\section{RNA extraction and purification}

RNA was extracted using the PicoPure RNA extraction kit (Thermo Fisher Scientific, Waltham, Massachusetts, USA). Due to the low amount of astrocytic RNA messured by Qubit (RNA high sensitivity assay, Thermo Fisher Scientific, Waltham, Massachusetts, USA) derived from as little as $0.1 \mathrm{gr}$ of brain slices, amplification was needed. In order to amplify full length cDNA transcripts Smart-seq principals were used. Therefore, the reverse transcription reaction was performed using a mix of different anchored VN-primer as well as template switching oligo with SuperScript III (Thermo Fisher Scientific, Carlsbad, USA). Input RNA and enzymes were mixed according to the manufacturers protocol. After RT-primer annealing template switching oligo (....) was added. The sample was placed on a thermal cycler running the following program: $25^{\circ} \mathrm{C}$ for $10 \mathrm{~min}, 50^{\circ} \mathrm{C}$ for $50 \mathrm{~min}$, followed by $42^{\circ} \mathrm{C}$ for $10 \mathrm{~min}$ and $85^{\circ} \mathrm{C}$ for $5 \mathrm{~min}$. In order to remove contaminating RNA, $1 \mu$ l of RNAse $\mathrm{H}$ (Thermo Fisher Scientific, Waltham, Massachusetts, USA) is added and incubated at $37^{\circ} \mathrm{C}$ for $20 \mathrm{~min}$. The resulting cDNA concentration post-reverse transcription is analyzed via Qubit (High sensitivity ssDNA assay). To amplify resulting 
cDNA a limited cycle PCR with a maximum of 10 cycles is performed. Primers complementary to the template switching oligo and to the reverse transcription VN-primers were added. Amplification progress was monitored by Evagreen dye (Biotium, Fremont, USA). After amplification Kappa pure beads (Roche Applied Science, Penzberg, Germany) are used for size selection $(0,8 \mathrm{x})$, excluding DNA fragments smaller then 300 base pairs. Subsequent DNA concentration is once again measured by Qubit analysis (High sensitivity dsDNA assay).

\section{RNA sequencing}

501 Purified cDNA from the amplification steps were prepared for sequencing using Nextera XT library preparation kit (Illumina, San Diego, USA). Input cDNA quantities are previously normalized to a starting concentration of $0,2 \mathrm{ng} / \mu \mathrm{l}$. For each sample a unique combination of i5 and i7 indexes was used. After tagmentation and amplification, libraries are once more cleaned using Kappa pure beads (Roche Applied Science, Penzberg, Germany). Fragments with a length of over $300 \mathrm{bp}$ are selected. Using Qubits high sensitivity dsDNA Kit (Thermo) and the results of an Agilent bioanalyzer high sensitivity run, libraries are normalized to the concentration of $1 \mathrm{nM}$ and subsequently pooled. Sequencing was performed on Illumina Nextseq 550 using a high output 150 cycle Flow cell v2.5 (Illumina, San Diego, USA). The sequencing run was set up as an paired end run, splitting the number of cycles equally at 75 each between read one and two.

\section{Analysis of RNA-seq:}

513 BCL files were demultiplexed and converted into fastq files using the bcl2fastq converter (Illumina). We 514 aligned the *.fastq files by the STAR aligner (STAR 2.7.9a, https://github.com/alexdobin/STAR, reference genome: GRCh38) resulting in a count matrix. Count data were processed by the RNA-seq pipeline (VisLab) as part of our in-house toolbox (https://github.com/heilandd/Vis_Lab1.5). Analysis of differentially expressed genes was performed using the DESeq2 package (Love et al., 2014). The algorithm mainly uses a generalized linear model with a negative binomial distribution. A detailed description is given in the $\mathrm{R}$ documentation.

\section{Gene expression analysis of multiple groups and clustering:}

522 Clustering and validation was performed as described recently (Henrik Heiland et al., 2019). First, we 523 calculated the optimal number of clusters based on the "Partitioning Around Medoids" algorithm. A t524 distributed stochastic neighbor embedding (tSNE) (Van der Maaten and Hinton, 2008) analysis and 525 Consensus Cluster (Wilkerson and Hayes, 2010) was performed to confirm the chosen k. Next, we used 526 either the PAMR (Hastie et al.) algorithm, a machine-learning based method, or a generalized linear 527 model (McCullagh, 1984) to identify characteristic up/downregulated genes in each subgroup. The 528 PAMR-score or the predictive-score extracted from the generalized linear model was used to identify 529 subclass specific gene set enrichment. Pipeline and codes are available at 530 https://github.com/heilandd/AutoPipe and CRAN AutoPipe, all analysis was described recently (Henrik 531 Heiland et al., 2019). 
535 For functional analysis, a permutation-based pre-ranked Gene Set Enrichment Analysis (GSEA) was

536 applied. We used predefined gene sets downloaded from the Molecular Signature Database v7.1. The

537 enrichment score of either differentially expressed genes (DE) or marker genes of clusters was

538 calculated by the rank order of gene expression/fold change DE. For significant enrichment, $p$-values

539 were adjusted by FDR. Gene Set Variation Analysis (GSVA) was performed with the GSVA package

540 implemented in R-software (Subramanian et al., 2005).

541

$542 \quad$ Integration of spatial transcriptomics

543 To project our extracted gene signatures into space, we imported the Visium 10X datasets available

544 from Ravi., et al 2021 including two IDH wild-type and two IDH mutated patients. The signature genes

545 from the above defined clusters of glioma-associated astrocytes were used to fit a non-negative matrix

546 factorization (NMF) (Elosua et al., 2020) implemented into the spotlight package. Surface plots were

547 generated using the SPATA2 software tool (https://github.com/theMILOlab/SPATA2).

548

549 Single-Cell RNA sequencing analysis

550 Single cell RNA-seq were downloaded from the Gene Expression Omnibus under GSE166418 (human

551 CEL-seq2 data). Postprocessing was performed by the MILO-pipeline for scRNA-seq

552 (https://github.com/theMILOlab/scPipelines). Single cell analysis was performed by the Seuratv4.0

553 package and SPATA 1.0 package. After pre-processing of the data through Seurat, we imported the

554 data into SPATA. Further analysis was performed as explained in the sections above.

555

556 Deep-learning integration of scRNA-seq and cell-type RNA-sequencing

557 To integrate RNA-sequencing from purified microglia into the latent space of the scRNA-seq, we trained 558 a neural network to predict the first two eigenvectors based on gene expression. The trained model was 559 used to fit our RNA-sequencing (microglia bulk) into the scRNA-seq latent space.

560

561

Immunofluorescence and Imaging

562 Immunostaining of slices with or without microglia and tumour injection was performed following the

563 established protocol. Growth media was removed and exchanged for $1 \mathrm{ml}$ of $4 \%$ paraformaldehyde

564 (PFA) for $1 \mathrm{~h}$ and further incubated in $20 \%$ methanol in PBS for $5 \mathrm{~min}$. Slices were then permeabilized

565 by incubating in PBS supplemented with $1 \%$ Triton (TX-100) overnight at $4{ }^{\circ} \mathrm{C}$ and further blocked using

$56620 \%$ BSA for $4 \mathrm{~h}$. The permeabilized and blocked slices were then incubated in the following primary

567 antibodies: anti- NeuN rabbit for neuronal nuclei (1:1000), anti-GFAP chicken for astrocytes (1:2000),

568 Iba-1 (1:1000, pig) for microglia. After washing in PBS, slices were labelled with secondary antibodies 569 conjugated with Alexa 488, Alexa 555 and Alexa 647 for $3 \mathrm{~h}$ at room temperature. Slices were then 570 mounted on glass slides additionally using DAPI fluoromount (Southern Biotech, Cat. No. 0100-20).

571 Fluorescence imaging was performed using a Fluoview FV10i confocal microscope from Olympus. All

572 measurements and image processing were performed using the company's software. Optical 573 magnification settings of $\times 10$ and $\times 60$ with oil were used. 
575 Separation and detection of metabolites. This step was performed as previously reported (Maier et al., 576 2021; Xu et al., 2018), with modifications. Metabolites were separated on an AC 3 AQ column, $1.0 \mathrm{x}$ $577150 \mathrm{~mm}$ (HiChrom) using a gradient of solvents $A(0.1 \%$ formic acid in water) and $B(0.1 \%$ formic acid 578 in $\mathrm{MeOH}$ ) over 10 minutes as follows: $0-0.5 \min 15 \%$ B, 0.5-5 min $85 \%$ B, 5-6 min $85 \%$ B, 6-6.20 min $57915 \%$ B, 6.21-10 min $15 \%$ B. The flow rate was $0.050 \mathrm{~mL} / \mathrm{min}$ connected to a Nexera X2 LC system 580 (Shimadzu). Metabolites were detected on a Sciex 6500+ ESI-tripleQ MS/MS on low mass mode (0$5811000 \mathrm{Da}$ ), with curtain gas (CUR) at 40, collision gas (CAD) at 10, lon spray voltage (IS) at 5000 Volts, 582 temperature (TEM) at $400^{\circ} \mathrm{C}$, ion source gas 1 (GS1) at 40 and ion source gas 2 (GS2) at 30 . Each 583 metabolite was optimized individually using chromatographic solvent conditions and exhibited optimal 584 ionization in the positive mode (Table 1).

585

586

587

588

589

590

591

592

593

594

595

\section{Table 1. Mass transitions and optimized mass spectrometry parameters for the neurotransmitters and internal standards in this study.}

\begin{tabular}{|c|c|c|c|c|c|c|c|}
\hline Q1 & Q3 & $\begin{array}{c}\text { Dwelling } \\
\text { time (ms) }\end{array}$ & Metabolite & DP & EP & CE & CXP \\
\hline 177,1 & 160,2 & 20 & Serotonine (5-HT) & 38 & 10 & 10 & 7 \\
\hline 148,2 & 84,1 & 20 & Glutamate (Glu) & 41 & 10 & 24 & 15 \\
\hline 104,2 & 87,1 & 20 & GABA $(\gamma$-aminobutyric & 26 & 14 & 15 & 16 \\
& & acid) & & & & \\
\hline 154,1 & 137,2 & 20 & Dopamine (DA) & 37 & 10 & 13 & 8 \\
\hline 205,2 & 188 & 20 & Tryptophan & 40 & 4 & 14 & 12 \\
\hline 182,2 & 136,1 & 20 & Tyrosine & 52 & 8 & 25 & 7 \\
\hline 212 & 152,2 & 20 & Isoprenaline & 46 & 8 & 20 & 9 \\
\hline 76,1 & 30 & 20 & Glycine (Gly) & 6 & 7,6 & 19 & 14 \\
\hline 158,1 & 141,2 & 20 & D4-Dopamine & 37 & 10 & 13 & 8 \\
\hline 106,1 & 60 & 20 & Serine (Ser) & 6 & 10,5 & 15,5 & 7 \\
\hline 78,1 & 31 & 20 & ${ }^{13}$ C, ${ }^{15}$ N-Gly & 6 & 7,6 & 19 & 14 \\
\hline 105,048 & 59,1 & 20 & Choline & 76 & 10 & 39 & 8 \\
\hline 162,055 & 98,1 & 20 & L-2-AAA & 56 & 10 & 21 & 10 \\
\hline
\end{tabular}

Calibrators and internal standards: All metabolites were quantified with respect to a calibration curve and selected internal standards. The dynamic range of calibrators was 0-5 $\mu \mathrm{M}$ for Glutamate, Serine, Dopamine, Serotonin and GABA, and 0-100 $\mu \mathrm{M}$ for Glycine, Tryptophan, Tyrosine, Choline and 2-Laminoadipic acid. D4- dopamine was utilized as internal standard for the quantification of dopamine. Glycine-1-13 $\mathrm{C},{ }^{15} \mathrm{~N}$ was utilized as internal standard for the quantification of Glycine. All other metabolites were normalized by isoprenaline as the internal standard, as described in a published method validated according to FDA guidelines (REF). The internal standard mix contained D4-dopamine (5 $\mu \mathrm{M})$, Glycine-

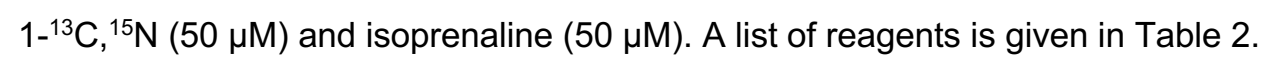




\begin{tabular}{|l|l|l|}
\hline \multicolumn{3}{|l|}{ Table 2. List of reagents, supplier and product numbers } \\
\hline Reagent & Supplier & Product number \\
\hline D-Serine & Sigma & S4250-5G \\
\hline L-Serine & Sigma & S4500-1G \\
\hline Dopamine & Sigma & H8502-5G \\
\hline Dopamine-D4 hydrochloride solution & Sigma & D-072-1ML \\
\hline Serotonin hydrochloride & Sigma & H9523-100MG \\
\hline GABA, g-amino butyric acid & Sigma & A2129-10G \\
\hline L-Glutamate & Sigma & $49621-250 G$ \\
\hline Glycine hydrochloride & Sigma & G2879-100G \\
\hline Glycine-1-13C, ${ }^{15} \mathrm{~N}$ & Sigma & $299340-100 M G$ \\
\hline L-2-Aminoadipic acid & Sigma & A7275-250MG \\
\hline Tryptophan & Sigma & T8941-25G \\
\hline Isoprenaline & Sigma & I5627-5G \\
\hline Water, CHROMASOLV ${ }^{\text {TM }}$ LC-MS & $\begin{array}{l}\text { Honeywell Research } \\
\text { Chemicals }\end{array}$ & $39253-1 \mathrm{~L}$ \\
\hline $\begin{array}{l}\text { Methanol, CHROMASOLV TM LC-MS Ultra, tested } \\
\text { for UHPLC-MS }\end{array}$ & $\begin{array}{l}\text { Honeywell Research } \\
\text { Chemicals }\end{array}$ & $14262-1 \mathrm{~L}$ \\
\hline AC 3 AQ column 1.0 x 150 mm & HiChrom & ACE-116-1501 \\
\hline
\end{tabular}

597 Sample handling-Culture medium: Culture media was collected and centrifuged at $9447 \times g$ at room 598 temperature for $10 \mathrm{~min}$. The cleared culture media samples were stored at $-80^{\circ} \mathrm{C}$ for further metabolomic 599 analysis.

600 Sample handling-Tissue biopsies: Flash-Frozen tissue biopsies were stored at $-80^{\circ} \mathrm{C}$ until used. Frozen 601 tissue was quickly weighed before thawing on ice and resuspended with ice-cold lysis buffer (10 mL 602 PBS $+100 \mu$ l protease inhibitor cocktail (P8340-5ML, Sigma)) to a ratio of $100 \mathrm{mg}$ tissue per $\mathrm{mL}$ of lysis 603 buffer. The tissue was homogenized on ice with a tissue disruptor (47747-370, VWR). An aliquot of this 604 extract was diluted 1:10 with lysis buffer and utilized for metabolomics analysis and protein quantification 605 by the bicinchoninic acid assay (23225, Thermofisher).

606 Sample preparation: Neurotransmitters were extracted according to a fast acidic methanol extraction 607 procedure previously described for polar metabolite extraction (Behringer et al., 2019). Briefly, $20 \mu \mathrm{L}$ of 608 calibrator or biological sample of interest was mixed with $20 \mu \mathrm{L}$ of internal standard mix and $20 \mu \mathrm{L}$ of $6090.1 \%$ formic acid in $\mathrm{H}_{2} \mathrm{O}$. Metabolites were extracted by addition of $100 \mu \mathrm{L}$ of $0.1 \%$ formic acid in $\mathrm{MeOH}$. 610 Samples were vortexed, then centrifuged at $9447 \times g$ at room temperature for 10 minutes.

611 Data analysis: Quantification of metabolites was carried out with Analyst ${ }^{\circledR} 1.6 .3$ software, 2015 AB 612 Sciex.

\section{9. Extracellular electrophysiological recording experiments}

615 Recordings: Extracellular recordings were made as previously described (Ravi et al., 2019). Briefly, 616 recordings were made using an MEA 1060 UP (Multichannel Systems, Reutlingen). Sections were 617 placed onto the recording electrode (60PedotMEA200/30iR-Au-gr, Multichannel Systems, Reutlingen). 
618 A slice grid (HD5, Ala Scientific) was further placed over the section to ensure optimal contact with the 619 electrode array. Recordings were carried out post stabilization $(20 \mathrm{~min}$ ) for a duration of $20 \mathrm{mins}$. Data 620 was sampled at $10 \mathrm{kHz}$, at $37^{\circ} \mathrm{C}$ (TC02, Multichannel Systems, Reutlingen). For experiments involving 621 the usage of multiple compounds, a peristaltic pump (PPS2, Multichannel Systems, Reutlingen) was 622 used at a flow rate of $3 \mathrm{~mL} / \mathrm{min}$. All recordings were carried out in the same culture medium as described 623 above.

624 Analysis: Raw data was analyzed using the MC_Rack software (Multichannel Systems, Reutlingen). 625 Recordings were either high-pass filtered $(300 \mathrm{~Hz}-3000 \mathrm{~Hz})$ for neuronal activity or low pass filtered (0$626150 \mathrm{~Hz}$ ) to detect changes in local field potentials (LFP). For neuronal activity, the threshold was set to $6274.5 \times$ S.D. of the recorded signal. Data was further exported to the R computing environment for plotting 628 and analysis.

629 